Commun. Korean Math. Soc. 26 (2011), No. 2, pp. 169-182

DOI 10.4134/CKMS.2011.26.2.169

\title{
BIPOLAR FUZZY HYPER MV-DEDUCTIVE SYSTEMS OF HYPER MV-ALGEBRAS
}

\author{
Min Su Kang
}

\begin{abstract}
The notions of bipolar fuzzy hyper MV-subalgebras, (weak) bipolar fuzzy hyper MV-deductive system and precisely weak bipolar fuzzy hyper MV-deductive system are introduced, and their relations are investigated. Characterizations of bipolar fuzzy hyper MV-subalgebras and weak bipolar fuzzy hyper MV-deductive systems are provided.
\end{abstract}

\section{Introduction}

MV-algebras introduced by C. C. Chang [1] in 1958 provide an algebraic proof of completeness theorem of infinite valued Lukasewicz propositional calculus. The hyper structure theory was introduced by F. Marty [12] at the 8 th congress of Scandinavian Mathematicians in 1934. Since then many researches have worked in these areas. Recently in [5], Sh. Ghorbani, A. Hasankhni and E. Eslami applied the hyper structure to MV-algebras and introduced the concept of a hyper MV-algebra which is a generalization of an MV-algebra and investigated some related results. Based on [5, 4], L. Torkzadeh and A. Ahadpanah [13] discussed hyper MV-ideals in hyper MV-algebras. Jun et al. [6] introduced the notions of (weak) hyper MV-deductive systems and (weak) implicative hyper MV-deductive systems, and investigated several properties. They also discussed relations among hyper MV-deductive systems, weak hyper MV-deductive systems, implicative hyper MV-deductive systems and weak implicative hyper MV-deductive systems. Fuzzy set theory is established in the paper [14]. In the traditional fuzzy sets, the membership degrees of elements range over the interval $[0,1]$. The membership degree expresses the degree of belongingness of elements to a fuzzy set. The membership degree 1 indicates that an element completely belongs to its corresponding fuzzy set, and the membership degree 0 indicates that an element does not belong to the fuzzy set. The membership degrees on the interval $(0,1)$ indicate the partial membership to the fuzzy set. Sometimes, the membership degree means the

Received February 17, 2010.

2010 Mathematics Subject Classification. 06F35, 03G25.

Key words and phrases. bipolar fuzzy hyper MV-subalgebra, (weak) bipolar fuzzy hyper MV-deductive system, precisely weak bipolar fuzzy hyper MV-deductive system. 
satisfaction degree of elements to some property or constraint corresponding to a fuzzy set (see $[2,15])$. In the viewpoint of satisfaction degree, the membership degree 0 is assigned to elements which do not satisfy some property. The elements with membership degree 0 are usually regarded as having the same characteristics in the fuzzy set representation. By the way, among such elements, some have irrelevant characteristics to the property corresponding to a fuzzy set and the others have contrary characteristics to the property. The traditional fuzzy set representation cannot tell apart contrary elements from irrelevant elements. Only with the membership degrees ranged on the interval $[0,1]$, it is difficult to express the difference of the irrelevant elements from the contrary elements in fuzzy sets. If a set representation could express this kind of difference, it would be more informative than the traditional fuzzy set representation. Based on these observations, Lee [10] introduced an extension of fuzzy sets named bipolar-valued fuzzy sets. Using the the notion of bipolarvalued fuzzy set, Jun and Song [8] and Lee [9] discussed subalgebras and ideals of BCK/BCI-algebras based on bipolar-valued fuzzy sets. Jun et al. [7] considered extensions and translations of ideals in BCK/BCI-algebras based on bipolar fuzzy set theory.

In this paper, we apply the bipolar fuzzy set theory to the notions of hyper MV-subalgebras and (weak) hyper MV-deductive systems. We introduce the concepts of bipolar fuzzy hyper MV-subalgebras, (weak) bipolar fuzzy hyper MV-deductive systems and previously weak bipolar fuzzy hyper MV-deductive systems, and investigate their relations/properties. We provide characterizations of bipolar fuzzy hyper MV-subalgebras and weak bipolar fuzzy hyper MV-deductive systems.

\section{Preliminaries}

\subsection{Basic results on hyper MV-algebras}

A hyper $M V$-algebra is a nonempty set $M$ endowed with a hyper operation " $\oplus$ ", a unary operation " $*$ " and a constant " 0 " satisfying the following axioms:

(a1) $x \oplus(y \oplus z)=(x \oplus y) \oplus z$,

(a2) $x \oplus y=y \oplus x$,

(a3) $\left(x^{*}\right)^{*}=x$

(a4) $\left(x^{*} \oplus y\right)^{*} \oplus y=\left(y^{*} \oplus x\right)^{*} \oplus x$,

(a5) $0^{*} \in x \oplus 0^{*}$,

(a6) $0^{*} \in x \oplus x^{*}$,

(a7) $x \ll y, y \ll x \Rightarrow x=y$

for all $x, y, z \in M$, where $x \ll y$ is defined by $0^{*} \in x^{*} \oplus y$.

For every subsets $A$ and $B$ of $M$, we define

$$
\begin{gathered}
A \ll B \Leftrightarrow(\exists a \in A)(\exists b \in B)(a \ll b), \\
A \oplus B=\bigcup_{a \in A, b \in B} a \oplus b .
\end{gathered}
$$


We also define $0^{*}=1$ and $A^{*}=\left\{a^{*} \mid a \in A\right\}$.

Every hyper MV-algebra $M$ satisfies the following assertions:

(b1) $(A \oplus B) \oplus C=A \oplus(B \oplus C)$,

(b2) $0 \ll x, \quad x \ll 1$,

(b3) $x \ll x$,

(b4) $x \ll y \Rightarrow y^{*} \ll x^{*}$,

(b5) $A \ll B \Rightarrow B^{*} \ll A^{*}$,

(b6) $A \ll A$,

(b7) $A \subseteq B \Rightarrow A \ll B$,

(b8) $x \ll x \oplus y, \quad A \ll A \oplus B$,

(b9) $\left(A^{*}\right)^{*}=A$,

(b10) $0 \oplus 0=\{0\}$,

(b11) $x \in x \oplus 0$,

(b12) $y \in x \oplus 0 \Rightarrow y \ll x$,

(b13) $y \oplus 0=x \oplus 0 \Rightarrow x=y$

for all $x, y, z \in M$ and subsets $A, B$ and $C$ of $M$.

A nonempty subset $S$ of a hyper MV-algebra $M$ is called a hyper $M V$ subalgebra of $M$ if $S$ is a hyper MV-algebra under the hyper operation " $\oplus$ " and the unary operation "*" on $M$.

Definition 2.1 ([6]). A nonempty subset $D$ of $M$ is called a weak hyper $M V$ deductive system of $M$ if it satisfies:

(i) $0 \in D$,

(ii) $(\forall x, y \in M)\left(\left(x^{*} \oplus y\right)^{*} \subseteq D, y \in D \Rightarrow x \in D\right)$.

Definition $2.2([6])$. A nonempty subset $D$ of $M$ is called a hyper $M V$ deductive system of $M$ if it satisfies Definition 2.1(i) and

$$
(\forall x, y \in M)\left(\left(x^{*} \oplus y\right)^{*} \ll D, y \in D \Rightarrow x \in D\right) .
$$

Note that every hyper MV-deductive system is a weak hyper MV-deductive system, but the converse is not true (see [6, Theorem 3.10 and Example 3.11]).

\subsection{Basic results on bipolar fuzzy sets}

Fuzzy sets are a kind of useful mathematical structure to represent a collection of objects whose boundary is vague. There are several kinds of fuzzy set extensions in the fuzzy set theory, for example, intuitionistic fuzzy sets, interval-valued fuzzy sets, vague sets etc. Bipolar-valued fuzzy sets are an extension of fuzzy sets whose membership degree range is enlarged from the interval $[0,1]$ to $[-1,1]$. Bipolar-valued fuzzy sets have membership degrees that represent the degree of satisfaction to the property corresponding to a fuzzy set and its counter-property. In a bipolar-valued fuzzy set, the membership degree 0 means that elements are irrelevant to the corresponding property, the membership degrees on $(0,1]$ indicate that elements somewhat satisfy the property, and the membership degrees on $[-1,0)$ indicate that elements somewhat satisfy 
the implicit counter-property (see [10]). Let $X$ be the universe of discourse. A bipolar-valued fuzzy set $f$ in $X$ is an object having the form

$$
f=\left\{\left(x, f_{n}(x), f_{p}(x)\right) \mid x \in X\right\},
$$

where $f_{n}: X \rightarrow[-1,0]$ and $f_{p}: X \rightarrow[0,1]$ are mappings. The positive membership degree $f_{p}(x)$ denotes the satisfaction degree of an element $x$ to the property corresponding to a bipolar-valued fuzzy set $f=\left\{\left(x, f_{n}(x), f_{p}(x)\right)\right.$ | $x \in X\}$, and the negative membership degree $f_{n}(x)$ denotes the satisfaction degree of $x$ to some implicit counter-property of $f=\left\{\left(x, f_{n}(x), f_{p}(x)\right) \mid x \in X\right\}$. If $f_{p}(x) \neq 0$ and $f_{n}(x)=0$, it is the situation that $x$ is regarded as having only positive satisfaction for $f=\left\{\left(x, f_{n}(x), f_{p}(x)\right) \mid x \in X\right\}$. If $f_{p}(x)=0$ and $f_{n}(x) \neq 0$, it is the situation that $x$ does not satisfy the property of $f=\left\{\left(x, f_{n}(x), f_{p}(x)\right) \mid x \in X\right\}$ but somewhat satisfies the counter-property of $f=\left\{\left(x, f_{n}(x), f_{p}(x)\right) \mid x \in X\right\}$. It is possible for an element $x$ to be $f_{p}(x) \neq 0$ and $f_{n}(x) \neq 0$ when the membership function of the property overlaps that of its counter-property over some portion of the domain (see [11]). For the sake of simplicity, we shall use the symbol $f=\left(M ; f_{n}, f_{p}\right)$ for the bipolar-valued fuzzy set $f=\left\{\left(x, f_{n}(x), f_{p}(x)\right) \mid x \in X\right\}$, and use the notion of bipolar fuzzy sets instead of the notion of bipolar-valued fuzzy sets.

\section{Bipolar fuzzy hyper MV-deductive systems}

In what follows, let $M$ denote a hyper MV-algebra unless otherwise specified. For any family $\left\{a_{i} \mid i \in \Lambda\right\}$ of real numbers, we define

$$
\begin{aligned}
& \vee\left\{a_{i} \mid i \in \Lambda\right\}:= \begin{cases}\max \left\{a_{i} \mid i \in \Lambda\right\} & \text { if } \Lambda \text { is finite, } \\
\sup \left\{a_{i} \mid i \in \Lambda\right\} & \text { otherwise, }\end{cases} \\
& \wedge\left\{a_{i} \mid i \in \Lambda\right\}:= \begin{cases}\min \left\{a_{i} \mid i \in \Lambda\right\} & \text { if } \Lambda \text { is finite, } \\
\inf \left\{a_{i} \mid i \in \Lambda\right\} & \text { otherwise. }\end{cases}
\end{aligned}
$$

Moreover, if $\Lambda=\{1,2, \ldots, n\}$, then $\vee\left\{a_{i} \mid i \in \Lambda\right\}$ and $\wedge\left\{a_{i} \mid i \in \Lambda\right\}$ are denoted by $a_{1} \vee a_{2} \vee \cdots \vee a_{n}$ and $a_{1} \wedge a_{2} \wedge \cdots \wedge a_{n}$, respectively.

For a bipolar fuzzy set $f=\left(M ; f_{n}, f_{p}\right)$ and $(\alpha, \beta) \in[-1,0] \times[0,1]$, we define

$$
\begin{aligned}
& N(f ; \alpha):=\left\{x \in X \mid f_{n}(x) \leq \alpha\right\}, \\
& P(f ; \beta):=\left\{x \in X \mid f_{p}(x) \geq \beta\right\}
\end{aligned}
$$

which are called the negative $\alpha$-cut of $f=\left(M ; f_{n}, f_{p}\right)$ and the positive $\beta$-cut of $f=\left(M ; f_{n}, f_{p}\right)$, respectively. The set

$$
C(f ;(\alpha, \beta)):=N(f ; \alpha) \cap P(f ; \beta)
$$

is called the $(\alpha, \beta)$-cut of $f=\left(M ; f_{n}, f_{p}\right)$. For every $k \in(0,1)$, if $(\alpha, \beta)=$ $(-k, k)$, then the set

$$
C(f ; k):=N(f ;-k) \cap P(f ; k)
$$

is called the $k$-cut of $f=\left(M ; f_{n}, f_{p}\right)$. 
TABLE 1. $\oplus$-multiplication and unary operation

\begin{tabular}{l|lll}
\hline$\oplus$ & 0 & $a$ & 1 \\
\hline 0 & $\{0\}$ & $\{a\}$ & $\{1\}$ \\
$a$ & $\{a\}$ & $\{0, a, 1\}$ & $\{0, a, 1\}$ \\
1 & $\{1\}$ & $\{0, a, 1\}$ & $\{1\}$ \\
\hline
\end{tabular}

\begin{tabular}{l|l}
\hline$x$ & $x^{*}$ \\
\hline 0 & 1 \\
$a$ & $a$ \\
1 & 0 \\
\hline
\end{tabular}

Definition 3.1. A bipolar fuzzy set $f=\left(M ; f_{n}, f_{p}\right)$ in $M$ is called a bipolar fuzzy hyper $M V$-subalgebra of $M$ if it satisfies the following assertions:

(i) $(\forall x, y \in M)\left(f_{n}\left(x^{*}\right) \leq f_{n}(x), f_{p}\left(y^{*}\right) \geq f_{p}(y)\right)$,

(ii) $(\forall x, y, u, v \in M)\left(\begin{array}{c}\bigvee_{a \in x \oplus y} f_{n}(a) \leq f_{n}(x) \vee f_{n}(y), \\ \bigwedge_{b \in u \oplus v} f_{p}(b) \geq f_{p}(u) \wedge f_{p}(v)\end{array}\right)$.

Example 3.2. Let $M=\{0, a, 1\}$ be a set with the hyper operation " $\oplus$ " and the unary operation "*" which are given by Table 1 . Then $\left(M, \oplus,{ }^{*}, 0\right)$ is a hyper MV-algebra (see [3]). Let $f=\left(M ; f_{n}, f_{p}\right)$ be a bipolar fuzzy set in $M$ given by

\begin{tabular}{|r|rrr|}
\hline$M$ & 0 & $a$ & 1 \\
\hline \hline$f_{n}$ & -0.8 & -0.3 & -0.8 \\
\hline$f_{p}$ & 0.7 & 0.5 & 0.7 \\
\hline
\end{tabular}

By routine calculations, we know that $f=\left(M ; f_{n}, f_{p}\right)$ is a bipolar fuzzy hyper MV-subalgebra of $M$.

Proposition 3.3. Every bipolar fuzzy hyper $M V$-subalgebra $f=\left(M ; f_{n}, f_{p}\right)$ of $M$ satisfies the following inequality:

$$
(\forall x \in M)\left(f_{n}(x) \geq f_{n}(1), f_{p}(x) \leq f_{p}(1)\right) .
$$

Proof. Since $1=0^{*} \in x^{*} \oplus x$ for all $x \in M$, it follows from Definition 3.1 that

$$
\begin{gathered}
f_{n}(1) \leq \bigvee_{a \in x^{*} \oplus x} f_{n}(a) \leq f_{n}\left(x^{*}\right) \vee f_{n}(x) \leq f_{n}(x) \vee f_{n}(x)=f_{n}(x), \\
f_{p}(1) \geq \bigwedge_{a \in x^{*} \oplus x} f_{p}(a) \geq f_{p}\left(x^{*}\right) \wedge f_{p}(x) \geq f_{p}(x) \wedge f_{p}(x)=f_{p}(x)
\end{gathered}
$$

for all $x \in M$. This completes the proof.

Lemma 3.4 ([3]). A nonempty subset $S$ of $M$ is a hyper $M V$-subalgebra of $M$ if and only if $x^{*} \in S$ and $x \oplus y \subseteq S$ for all $x, y \in S$.

We provide a characterization of a bipolar fuzzy hyper MV-subalgebra.

Theorem 3.5. A bipolar fuzzy set $f=\left(M ; f_{n}, f_{p}\right)$ in $M$ is a bipolar fuzzy hyper $M V$-subalgebra of $M$ if and only if both the nonempty negative $\alpha$-cut and the nonempty positive $\beta$-cut of $f=\left(M ; f_{n}, f_{p}\right)$ are hyper $M V$-subalgebras of $M$ for all $(\alpha, \beta) \in[-1,0] \times[0,1]$. 
Proof. Assume that $f=\left(M ; f_{n}, f_{p}\right)$ is a bipolar fuzzy hyper MV-subalgebra of $M$. Let $N(f ; \alpha)$ and $P(f ; \beta)$ be the nonempty negative $\alpha$-cut and the nonempty positive $\beta$-cut of $f=\left(M ; f_{n}, f_{p}\right)$, respectively, for all $(\alpha, \beta) \in[-1,0] \times[0,1]$. Let $x \in P(f ; \beta)$ and $y \in N(f ; \alpha)$. Then $f_{p}(x) \geq \beta$ and $f_{n}(y) \leq \alpha$, and so $f_{p}\left(x^{*}\right) \geq f_{p}(x) \geq \beta$ and $f_{n}\left(y^{*}\right) \leq f_{n}(y) \leq \alpha$ by Definition 3.1(i). Thus $x^{*} \in P(f ; \beta)$ and $y^{*} \in N(f ; \alpha)$. For any $x, y \in N(f ; \alpha)$ and $u, v \in P(f ; \beta)$, let $z \in x \oplus y$ and $w \in u \oplus v$. Using Definition 3.1(ii), we have

$$
\begin{aligned}
& f_{n}(z) \leq \bigvee_{a \in x \oplus y} f_{n}(a) \leq f_{n}(x) \vee f_{n}(y) \leq \alpha, \\
& f_{p}(w) \geq \bigwedge_{b \in u \oplus v} f_{p}(b) \geq f_{p}(u) \wedge f_{p}(v) \geq \beta .
\end{aligned}
$$

Hence $z \in N(f ; \alpha)$ and $w \in P(f ; \beta)$. Thus $x \oplus y \subseteq N(f ; \alpha)$ and $u \oplus v \subseteq P(f ; \beta)$. Therefore $N(f ; \alpha)$ and $P(f ; \beta)$ are hyper MV-subalgebras of $M$ by Lemma 3.4.

Conversely, suppose that both the nonempty negative $\alpha$-cut and the nonempty positive $\beta$-cut of $f=\left(M ; f_{n}, f_{p}\right)$ are hyper MV-subalgebras of $M$ for all $(\alpha, \beta) \in[-1,0] \times[0,1]$. If there exists $a \in M$ such that $f_{p}\left(a^{*}\right)<f_{p}(a)$, then $f_{p}\left(a^{*}\right)<\beta_{a} \leq f_{p}(a)$ for some $\beta_{a} \in(0,1]$. It follows that $a \in P\left(f ; \beta_{a}\right)$ but $a^{*} \notin P\left(f ; \beta_{a}\right)$. This is a contradiction, and so $f_{p}\left(x^{*}\right) \geq f_{p}(x)$ for all $x \in M$. Suppose that there exists $b \in M$ such that $f_{n}\left(b^{*}\right)>f_{n}(b)$. Then $f_{n}(b) \leq \alpha_{b}<$ $f_{n}\left(b^{*}\right)$ for some $\alpha_{b} \in[-1,0)$. Thus $b \in N\left(f ; \alpha_{b}\right)$ but $b^{*} \notin N\left(f ; \alpha_{b}\right)$, which is a contradiction. Hence $f_{n}\left(y^{*}\right) \leq f_{n}(y)$ for all $y \in M$. For any $x, y \in M$, let $\beta:=f_{p}(x) \wedge f_{p}(y)$. Then $x, y \in P(f ; \beta)$, and thus $x \oplus y \subseteq P(f ; \beta)$ since $P(f ; \beta)$ is a hyper MV-subalgebra of $M$. It follows that $f_{p}(a) \geq \beta$ for all $a \in x \oplus y$ so that

$$
\bigwedge_{a \in x \oplus y} f_{p}(a) \geq \beta=f_{p}(x) \wedge f_{p}(y)
$$

If we take $\alpha:=f_{n}(x) \vee f_{n}(y)$ for any $x, y \in M$, then $x, y \in N(f ; \alpha)$. Since $N(f ; \alpha)$ is a hyper MV-subalgebra of $M$, it follows from Lemma 3.4 that $x \oplus y \subseteq$ $N(f ; \alpha)$ so that $f_{n}(b) \leq \alpha$ for all $b \in x \oplus y$. Thus

$$
\bigvee_{b \in x \oplus y} f_{n}(b) \leq \alpha=f_{n}(x) \vee f_{n}(y) .
$$

Therefore $f=\left(M ; f_{n}, f_{p}\right)$ is a bipolar fuzzy hyper MV-subalgebra of $M$.

Corollary 3.6. If a bipolar fuzzy set $f=\left(M ; f_{n}, f_{p}\right)$ in $M$ is a bipolar fuzzy hyper $M V$-subalgebra of $M$, then the $k$-cut of $f=\left(M ; f_{n}, f_{p}\right)$ is a hyper $M V$ subalgebra of $M$ for all $k \in(0,1)$.

Definition 3.7. A bipolar fuzzy set $f=\left(M ; f_{n}, f_{p}\right)$ in $M$ is called a weak bipolar fuzzy hyper $M V$-deductive system of $M$ if it satisfies the following conditions

(i) $(\forall x, y \in M)\left(f_{n}(x) \geq f_{n}(0), f_{p}(y) \leq f_{p}(0)\right)$. 
TABLE 2. $\oplus$-multiplication and unary operation

\begin{tabular}{l|lll}
\hline$\oplus$ & 0 & $a$ & 1 \\
\hline 0 & $\{0\}$ & $\{0, a\}$ & $\{0,1\}$ \\
$a$ & $\{0, a\}$ & $\{0, a, 1\}$ & $\{0, a, 1\}$ \\
1 & $\{0,1\}$ & $\{0, a, 1\}$ & $\{0,1\}$ \\
\hline
\end{tabular}

\begin{tabular}{l|l}
\hline$x$ & $x^{*}$ \\
\hline 0 & 1 \\
$a$ & $a$ \\
1 & 0 \\
\hline
\end{tabular}

(ii) $(\forall x, y, u, v \in M)\left(\begin{array}{l}f_{n}(x) \leq\left(\bigvee_{a \in\left(x^{*} \oplus y\right)^{*}} f_{n}(a)\right) \vee f_{n}(y), \\ f_{p}(u) \geq\left(\bigwedge_{b \in\left(u^{*} \oplus v\right)^{*}} f_{p}(b)\right) \wedge f_{p}(v)\end{array}\right)$.

Example 3.8. Let $M=\{0, a, 1\}$ be a set with the hyper operation " $\oplus$ " and the unary operation "*" which are given by Table 2 . Then $\left(M, \oplus,{ }^{*}, 0\right)$ is a hyper MV-algebra. Let $f=\left(M ; f_{n}, f_{p}\right)$ be a bipolar fuzzy set in $M$ given by

\begin{tabular}{|r|rrr|}
\hline$M$ & 0 & $a$ & 1 \\
\hline \hline$f_{n}$ & -0.8 & -0.6 & -0.2 \\
\hline$f_{p}$ & 0.7 & 0.5 & 0.3 \\
\hline
\end{tabular}

Then $f=\left(M ; f_{n}, f_{p}\right)$ is a weak bipolar fuzzy hyper MV-deductive system of M.

Example 3.9. Let $X=\{0, a, 1\}$ be a hyper MV-algebra which is given in Example 3.2. Let $f=\left(M ; f_{n}, f_{p}\right)$ be a bipolar fuzzy set in $M$ given by

\begin{tabular}{|r|rrr|}
\hline$M$ & 0 & $a$ & 1 \\
\hline \hline$f_{n}$ & -0.8 & -0.5 & -0.3 \\
\hline$f_{p}$ & 0.7 & 0.3 & 0.2 \\
\hline
\end{tabular}

Then $f=\left(M ; f_{n}, f_{p}\right)$ is not a weak bipolar fuzzy hyper MV-deductive system of $M$, since

$$
f_{n}(1)=-0.3>-0.5=\left(\bigvee_{b \in\left(1^{*} \oplus a\right)^{*}} f_{n}(b)\right) \vee f_{n}(a)
$$

and/or

$$
f_{p}(1)=0.2<0.3=\left(\bigwedge_{b \in\left(1^{*} \oplus a\right)^{*}} f_{p}(b)\right) \wedge f_{p}(a)
$$


Proposition 3.10. Every weak bipolar fuzzy hyper $M V$-deductive system $f=$ $\left(M ; f_{n}, f_{p}\right)$ of $M$ satisfies the following assertion.

$$
(\forall x, y, u, v \in M)\left(\begin{array}{l}
f_{n}\left(y^{*}\right) \leq\left(\bigvee_{a \in\left(x^{*} \oplus y\right)^{*}} f_{n}(a)\right) \vee f_{n}\left(x^{*}\right), \\
f_{p}\left(v^{*}\right) \geq\left(\bigwedge_{b \in\left(u^{*} \oplus v\right)^{*}} f_{p}(b)\right) \wedge f_{p}\left(u^{*}\right)
\end{array}\right) .
$$

Proof. It follows from (a2), (a3) and Definition 3.7(ii).

We provide a characterization of a weak bipolar fuzzy hyper MV-deductive system.

Theorem 3.11. A bipolar fuzzy set $f=\left(M ; f_{n}, f_{p}\right)$ in $M$ is a weak bipolar fuzzy hyper $M V$-deductive system of $M$ if and only if both the nonempty negative $\alpha$-cut and the nonempty positive $\beta$-cut of $f=\left(M ; f_{n}, f_{p}\right)$ are weak hyper $M V$ deductive systems of $M$ for all $(\alpha, \beta) \in[-1,0] \times[0,1]$.

Proof. Assume that $f=\left(M ; f_{n}, f_{p}\right)$ is a weak bipolar fuzzy hyper MV-deductive system of $M$. Let $N(f ; \alpha)$ and $P(f ; \beta)$ be the nonempty negative $\alpha$-cut and the nonempty positive $\beta$-cut of $f=\left(M ; f_{n}, f_{p}\right)$, respectively, for all $(\alpha, \beta) \in$ $[-1,0] \times[0,1]$. Then there exist $a \in N(f ; \alpha)$ and $b \in P(f ; \beta)$. Thus $f_{n}(a) \leq \alpha$ and $f_{p}(b) \geq \beta$, which imply from Definition 3.7(i) that $f_{n}(0) \leq f_{n}(a) \leq \alpha$ and $f_{p}(0) \geq f_{p}(b) \geq \beta$ so that $0 \in N(f ; \alpha)$ and $0 \in P(f ; \beta)$. Let $x, y, u, v \in M$ be such that $y \in N(f ; \alpha),\left(x^{*} \oplus y\right)^{*} \subseteq N(f ; \alpha), v \in P(f ; \beta)$, and $\left(u^{*} \oplus v\right)^{*} \subseteq$ $P(f ; \beta)$. Then $f_{n}(y) \leq \alpha, f_{n}(z) \leq \alpha$ for all $z \in\left(x^{*} \oplus y\right)^{*}, f_{p}(v) \geq \beta$ and $f_{p}(w) \geq \beta$ for all $w \in\left(u^{*} \oplus v\right)^{*}$. Hence

$$
f_{n}(x) \leq\left(\bigvee_{a \in\left(x^{*} \oplus y\right)^{*}} f_{n}(a)\right) \vee f_{n}(y) \leq \alpha
$$

and

$$
f_{p}(u) \geq\left(\bigwedge_{b \in\left(u^{*} \oplus v\right)^{*}} f_{p}(b)\right) \wedge f_{p}(v) \geq \beta .
$$

Therefore $x \in N(f ; \alpha)$ and $u \in P(f ; \beta)$ which shows that $N(f ; \alpha)$ and $P(f ; \beta)$ are weak hyper MV-deductive systems of $M$.

Conversely, suppose that both the nonempty negative $\alpha$-cut and the nonempty positive $\beta$-cut of $f=\left(M ; f_{n}, f_{p}\right)$ are weak hyper MV-deductive systems of $M$ for all $(\alpha, \beta) \in[-1,0] \times[0,1]$. Let

$$
\alpha:=\left(\bigvee_{a \in\left(x^{*} \oplus y\right)^{*}} f_{n}(a)\right) \vee f_{n}(y)
$$


and

$$
\beta:=\left(\bigwedge_{b \in\left(u^{*} \oplus v\right)^{*}} f_{p}(b)\right) \wedge f_{p}(v)
$$

for all $x, y, u, v \in M$. Then $y \in N(f ; \alpha)$ and $v \in P(f ; \beta)$. For each $c \in\left(x^{*} \oplus y\right)^{*}$ and $d \in\left(u^{*} \oplus v\right)^{*}$ we have

$$
f_{n}(c) \leq \bigvee_{a \in\left(x^{*} \oplus y\right)^{*}} f_{n}(a) \leq\left(\bigvee_{a \in\left(x^{*} \oplus y\right)^{*}} f_{n}(a)\right) \vee f_{n}(y)=\alpha
$$

and

$$
f_{p}(d) \geq \bigwedge_{b \in\left(u^{*} \oplus v\right)^{*}} f_{p}(b) \geq\left(\bigwedge_{b \in\left(u^{*} \oplus v\right)^{*}} f_{p}(b)\right) \wedge f_{p}(v)=\beta .
$$

It follows that $c \in N(f ; \alpha)$ and $d \in P(f ; \beta)$ so that $\left(x^{*} \oplus y\right)^{*} \subseteq N(f ; \alpha)$ and $\left(u^{*} \oplus v\right)^{*} \subseteq P(f ; \beta)$. Since $N(f ; \alpha)$ and $P(f ; \beta)$ are weak hyper MV-deductive systems of $M$, it follows from Definition 2.1(ii) that $x \in N(f ; \alpha)$ and $u \in P(f ; \beta)$ so that

and

$$
f_{n}(x) \leq \alpha=\left(\bigvee_{a \in\left(x^{*} \oplus y\right)^{*}} f_{n}(a)\right) \vee f_{n}(y)
$$

$$
f_{p}(u) \geq \beta=\left(\bigwedge_{b \in\left(u^{*} \oplus v\right)^{*}} f_{p}(b)\right) \wedge f_{p}(v) .
$$

Consequently, $f=\left(M ; f_{n}, f_{p}\right)$ is a weak bipolar fuzzy hyper MV-deductive system of $M$.

Corollary 3.12. If a bipolar fuzzy set $f=\left(M ; f_{n}, f_{p}\right)$ in $M$ is a weak bipolar fuzzy hyper $M V$-deductive system of $M$, then the $k$-cut of $f=\left(M ; f_{n}, f_{p}\right)$ is a hyper $M V$-deductive system of $M$ for all $k \in(0,1)$.

Definition 3.13. A bipolar fuzzy set $f=\left(M ; f_{n}, f_{p}\right)$ in $M$ is called a bipolar fuzzy hyper $M V$-deductive system of $M$ if it satisfies Definition 3.7(ii) and

$$
(\forall x, y \in M)\left(x \ll y \Rightarrow f_{n}(x) \leq f_{n}(y), f_{p}(x) \geq f_{p}(y)\right) .
$$

Example 3.14. Consider a MV-algebra $M$ which is given in Example 3.8. Let $f=\left(M ; f_{n}, f_{p}\right)$ be a bipolar fuzzy set in $M$ defined by

\begin{tabular}{|r|rrr|}
\hline$M$ & 0 & $a$ & 1 \\
\hline \hline$f_{n}$ & -0.9 & -0.5 & -0.3 \\
\hline$f_{p}$ & 0.7 & 0.3 & 0.2 \\
\hline
\end{tabular}

Then $f=\left(M ; f_{n}, f_{p}\right)$ is a bipolar fuzzy hyper MV-deductive system of $M$. 
Let $f=\left(M ; f_{n}, f_{p}\right)$ be a bipolar fuzzy hyper MV-deductive system of $M$. Since $0 \ll x$ for all $x \in M$, it follows from (3.4) that $f_{n}(0) \leq f_{n}(x)$ and $f_{p}(0) \geq f_{p}(x)$ for all $x \in M$. Hence every bipolar fuzzy hyper MV-deductive system of $M$ is a weak bipolar fuzzy hyper MV-deductive system of $M$. But the converse is not valid as seen in the following example.

Example 3.15. Let $M$ be a hyper MV-algebra in Example 3.8. Let $f=$ $\left(M ; f_{n}, f_{p}\right)$ be a bipolar fuzzy set in $M$ defined by

\begin{tabular}{|c|rrr|}
\hline$M$ & 0 & $a$ & 1 \\
\hline \hline$f_{n}$ & -0.9 & -0.2 & -0.4 \\
\hline$f_{p}$ & 0.7 & 0.3 & 0.5 \\
\hline
\end{tabular}

Then $f=\left(M ; f_{n}, f_{p}\right)$ is a weak bipolar fuzzy hyper MV-deductive system of $M$, but not a bipolar fuzzy hyper MV-deductive system of $M$ since $a \ll 1$ but $f_{n}(a)=-0.2>-0.4=f_{n}(1)$ and $/$ or $f_{p}(a)=0.3<0.5=f_{p}(1)$.

Theorem 3.16. Let $f=\left(M ; f_{n}, f_{p}\right)$ be a bipolar fuzzy set in $M$ such that $N(f ; \alpha) \neq \emptyset$ and $P(f ; \beta) \neq \emptyset$ for all $(\alpha, \beta) \in[-1,0] \times[0,1]$. If $N(f ; \alpha)$ and $P(f ; \beta)$ are hyper $M V$-deductive systems of $M$ for all $(\alpha, \beta) \in[-1,0] \times[0,1]$, then $f=\left(M ; f_{n}, f_{p}\right)$ is a bipolar fuzzy hyper $M V$-deductive system of $M$.

Proof. Let $x, y \in M$ be such that $x \ll y$. Taking $\alpha=f_{n}(y)$ and $\beta=f_{p}(y)$, we have $y \in N(f ; \alpha)$ and $y \in P(f ; \beta)$, and thus $x \in N(f ; \alpha)$ and $x \in P(f ; \beta)$ because $N(f ; \alpha)$ and $P(f ; \beta)$ are hyper MV-deductive systems of $M$. Hence $f_{n}(x) \leq \alpha=f_{n}(y)$ and $f_{p}(x) \geq \beta=f_{p}(y)$. For every $x, y, u, v \in M$, let

$$
\begin{aligned}
\alpha & :=\left(\bigvee_{a \in\left(x^{*} \oplus y\right)^{*}} f_{n}(a)\right) \vee f_{n}(y), \\
\beta & :=\left(\bigwedge_{b \in\left(u^{*} \oplus v\right)^{*}} f_{p}(b)\right) \wedge f_{p}(v) .
\end{aligned}
$$

Then $y \in N(f ; \alpha)$ and $v \in P(f ; \beta)$, and for each $c \in\left(x^{*} \oplus y\right)^{*}$ and $d \in\left(u^{*} \oplus v\right)^{*}$ we have

$$
f_{n}(c) \leq \bigvee_{a \in\left(x^{*} \oplus y\right)^{*}} f_{n}(a) \leq\left(\bigvee_{a \in\left(x^{*} \oplus y\right)^{*}} f_{n}(a)\right) \vee f_{n}(y)=\alpha
$$

and

$$
f_{p}(d) \geq \bigwedge_{b \in\left(u^{*} \oplus v\right)^{*}} f_{p}(b) \geq\left(\bigwedge_{b \in\left(u^{*} \oplus v\right)^{*}} f_{p}(b)\right) \wedge f_{p}(v)=\beta .
$$

Thus $c \in N(f ; \alpha)$ and $d \in P(f ; \beta)$, i.e., $\left(x^{*} \oplus y\right)^{*} \subseteq N(f ; \alpha)$ and $\left(u^{*} \oplus v\right)^{*} \subseteq$ $P(f ; \beta)$. It follows from $(\mathrm{b} 7)$ that $\left(x^{*} \oplus y\right)^{*} \ll N(f ; \alpha)$ and $\left(u^{*} \oplus v\right)^{*} \ll P(f ; \beta)$. 
Since $N(f ; \alpha)$ and $P(f ; \beta)$ are hyper MV-deductive systems of $M$, we obtain $x \in N(f ; \alpha)$ and $u \in P(f ; \beta)$ by $(2.1)$. Hence

$$
(\forall x, y, u, v \in M)\left(\begin{array}{l}
f_{n}(x) \leq \alpha=\left(\bigvee_{a \in\left(x^{*} \oplus y\right)^{*}} f_{n}(a)\right) \vee f_{n}(y), \\
f_{p}(u) \geq \beta=\left(\bigwedge_{b \in\left(u^{*} \oplus v\right)^{*}} f_{p}(b)\right) \wedge f_{p}(v)
\end{array}\right) .
$$

Therefore $f=\left(M ; f_{n}, f_{p}\right)$ is a bipolar fuzzy hyper MV-deductive system of $M$.

Definition 3.17. A bipolar fuzzy set $f=\left(M ; f_{n}, f_{p}\right)$ in $M$ is called a previously weak bipolar fuzzy hyper $M V$-deductive system of $M$ if it satisfies Definition 3.7(i) and for all $x, y, u, v \in M$ there exist $a \in\left(x^{*} \oplus y\right)^{*}$ and $b \in\left(u^{*} \oplus v\right)^{*}$ such that

$$
f_{n}(x) \leq f_{n}(a) \wedge f_{n}(y) \text { and } f_{p}(u) \geq f_{p}(b) \vee f_{p}(v) .
$$

Example 3.18. The weak bipolar fuzzy hyper MV-deductive system $f=$ $\left(M ; f_{n}, f_{p}\right)$ of $M$ in Example 3.8 is a previously weak bipolar fuzzy hyper MVdeductive system of $M$.

Theorem 3.19. Every previously weak bipolar fuzzy hyper $M V$-deductive system is a weak bipolar fuzzy hyper $M V$-deductive system.

Proof. Let $f=\left(M ; f_{n}, f_{p}\right)$ be a previously weak bipolar fuzzy hyper MVdeductive system of $M$ and let $x, y, u, v \in M$. Then there exist $a \in\left(x^{*} \oplus y\right)^{*}$ and $b \in\left(u^{*} \oplus v\right)^{*}$ such that

$$
f_{n}(x) \leq f_{n}(a) \wedge f_{n}(y) \text { and } f_{p}(u) \geq f_{p}(b) \vee f_{p}(v) .
$$

Note that $f_{n}(a) \leq \bigvee_{c \in\left(x^{*} \oplus y\right)^{*}} f_{n}(c)$ and $f_{p}(b) \geq \bigwedge_{d \in\left(u^{*} \oplus v\right)^{*}} f_{p}(d)$, and so

$$
f_{n}(x) \leq\left(\bigvee_{c \in\left(x^{*} \oplus y\right)^{*}} f_{n}(c)\right) \vee f_{n}(y)
$$

and

$$
f_{p}(u) \geq\left(\bigwedge_{d \in\left(u^{*} \oplus v\right)^{*}} f_{p}(d)\right) \wedge f_{p}(v) .
$$

Hence $f=\left(M ; f_{n}, f_{p}\right)$ is a weak bipolar fuzzy hyper MV-deductive system of $M$.

A bipolar fuzzy set $f=\left(M ; f_{n}, f_{p}\right)$ in $M$ is said to satisfy the sup-inf property if for any nonempty subset $T$ of $M$ there exist $x_{0}, y_{0} \in T$ such that $f_{n}\left(x_{0}\right)=$ $\bigvee_{x \in T} f_{n}(x)$ and $f_{p}\left(y_{0}\right)=\bigwedge_{y \in T} f_{p}(y)$. 
Theorem 3.20. Let $f=\left(M ; f_{n}, f_{p}\right)$ be a weak bipolar fuzzy hyper $M V$-deductive system of $M$. If $f=\left(M ; f_{n}, f_{p}\right)$ satisfies the sup-inf property, then $f=$ $\left(M ; f_{n}, f_{p}\right)$ is a previously weak bipolar fuzzy hyper $M V$-deductive system of $M$.

Proof. Since $f=\left(M ; f_{n}, f_{p}\right)$ satisfies the sup-inf property, there exist $a \in$ $\left(x^{*} \oplus y\right)^{*}$ and $b \in\left(u^{*} \oplus v\right)^{*}$ such that $f_{n}(a)=\bigvee_{c \in\left(x^{*} \oplus y\right)^{*}} f_{n}(c)$ and $f_{p}(b)=$ $\bigwedge_{d \in\left(u^{*} \oplus v\right)^{*}} f_{p}(d)$. It follows from Definition 3.7(ii) that

$$
f_{n}(x) \leq\left(\bigvee_{c \in\left(x^{*} \oplus y\right)^{*}} f_{n}(c)\right) \vee f_{n}(y)=f_{n}(a) \vee f_{n}(y)
$$

and

$$
f_{p}(u) \geq\left(\bigwedge_{d \in\left(u^{*} \oplus v\right)^{*}} f_{p}(d)\right) \wedge f_{p}(v)=f_{p}(b) \wedge f_{p}(v) .
$$

Hence $f=\left(M ; f_{n}, f_{p}\right)$ is a previously weak bipolar fuzzy hyper MV-deductive system of $M$.

Corollary 3.21. Every bipolar fuzzy hyper $M V$-deductive system satisfying the sup-inf property is a previously weak bipolar fuzzy hyper $M V$-deductive system.

Note that if a hyper MV-algebra $M$ is finite, then every bipolar fuzzy set in $M$ satisfies the sup-inf property. Hence we know that in a finite hyper MV-algebra $M$,

(1) the notion of weak bipolar fuzzy hyper MV-deductive system coincide with the notion of previously weak bipolar fuzzy hyper MV-deductive system,

(2) a bipolar fuzzy hyper MV-deductive system is a previously weak bipolar fuzzy hyper MV-deductive system.

Theorem 3.22. If $f=\left(M ; f_{n}, f_{p}\right)$ is a bipolar fuzzy hyper $M V$-deductive system of $M$ satisfying the sup-inf property, then both the nonempty negative $\alpha$ cut and the nonempty positive $\beta$-cut of $f=\left(M ; f_{n}, f_{p}\right)$ are hyper $M V$-deductive systems of $M$ for all $(\alpha, \beta) \in[-1,0] \times[0,1]$.

Proof. Let $N(f ; \alpha)$ and $P(f ; \beta)$ be the non-empty negative $\alpha$-cut and the nonempty positive $\beta$-cut of $f=\left(M ; f_{n}, f_{p}\right)$, respectively, for all $(\alpha, \beta) \in$ $[-1,0] \times[0,1]$. Then there exist $a \in N(f ; \alpha)$ and $b \in P(f ; \beta)$, and so $f_{n}(a) \leq \alpha$ and $f_{p}(b) \geq \beta$. Hence $f_{n}(0) \leq f_{n}(a) \leq \alpha$ and $f_{p}(0) \geq f_{p}(b) \geq \beta$, i.e., $0 \in N(f ; \alpha)$ and $0 \in P(f ; \beta)$. Let $x, y, u, v \in M$ be such that $\left(x^{*} \oplus y\right)^{*} \ll$ $N(f ; \alpha), y \in N(f ; \alpha),\left(u^{*} \oplus v\right)^{*} \ll P(f ; \beta)$, and $v \in P(f ; \beta)$. Then there exist $w \in\left(x^{*} \oplus y\right)^{*}$ and $z \in N(f ; \alpha)$ such that $w \ll z$; and there exist $r \in\left(u^{*} \oplus v\right)^{*}$ and $q \in P(f ; \beta)$ such that $r \ll q$. Note that $f=\left(M ; f_{n}, f_{p}\right)$ is a weak bipolar fuzzy hyper MV-deductive system of $M$ satisfying the sup-inf property. Thus 
$f=\left(M ; f_{n}, f_{p}\right)$ is a previously weak bipolar fuzzy hyper MV-deductive system of $M$ by Theorem 3.20. Using (3.4) and (3.5), we have

$$
f_{n}(x) \leq f_{n}(w) \vee f_{n}(y) \leq f_{n}(z) \vee f_{n}(y) \leq \alpha
$$

and

$$
f_{p}(u) \geq f_{p}(r) \wedge f_{p}(v) \geq f_{p}(q) \vee f_{p}(v) \geq \beta,
$$

and thus $x \in N(f ; \alpha)$ and $u \in P(f ; \beta)$. Therefore $N(f ; \alpha)$ and $P(f ; \beta)$ are hyper MV-deductive systems of $M$.

Corollary 3.23. If a bipolar fuzzy set $f=\left(M ; f_{n}, f_{p}\right)$ in $M$ is a bipolar fuzzy hyper $M V$-deductive system of $M$ satisfying the sup-inf property, then the $k$-cut of $f=\left(M ; f_{n}, f_{p}\right)$ is a hyper $M V$-deductive system of $M$ for all $k \in(0,1)$.

Using Theorems 3.16 and 3.22, we know that if $M$ is a finite hyper MValgebra, then a bipolar fuzzy set $f=\left(M ; f_{n}, f_{p}\right)$ in $M$ is a bipolar fuzzy hyper MV-deductive system of $M$ if and only if both the nonempty negative $\alpha$-cut and the nonempty positive $\beta$-cut of $f=\left(M ; f_{n}, f_{p}\right)$ are hyper MV-deductive systems of $M$ for all $(\alpha, \beta) \in[-1,0] \times[0,1]$.

\section{References}

[1] C. C. Chang, Algebraic analysis of many valued logics, Trans. Amer. Math. Soc. 88 (1958), 467-490.

[2] D. Dubois and H. Prade, Fuzzy Sets and Systems, Academic Press, Inc. [Harcourt Brace Jovanovich, Publishers], New York-London, 1980.

[3] Sh. Ghorbani, On hyper MV-algebras, Ph. D. thesis, Shahbid Bahonar University of Kerman 2007.

[4] Sh. Ghorbani, E. Eslami, and A. Hasankhani, Quotient hyper MV-algebras, Sci. Math. Jpn. 66 (2007), no. 3, 371-386.

[5] Sh. Ghorbani, A. Hasankhani, and E. Eslami, Hyper MV-algebras, Set-Valued Math. Appl. 1 (2008), 205-222.

[6] Y. B. Jun, M. S. Kang, and H. S. Kim, Hyper $M V$-deductive systems of hyper $M V$ algebras, Commun. Korean Math. Soc. (submitted).

[7] Y. B. Jun, S. Y. Kim, and E. H. Roh, Bipolar fuzzy trabslations and extensions of ideals in BCK/BCI-algebras, Tamkang J. Math. (submitted).

[8] Y. B. Jun and S. Z. Song, Subalgebras and closed ideals of BCH-algebras based on bipolar-valued fuzzy sets, Sci. Math. Jpn. 68 (2008), no. 2, 287-297.

[9] K. J. Lee, Bipolar fuzzy subalgebras and bipolar fuzzy ideals of BCK/BCI-algebras, Bull. Malays. Math. Sci. Soc. (2) 32 (2009), no. 3, 361-373.

[10] K. M. Lee, Bipolar-valued fuzzy sets and their operations, Proc. Int. Conf. on Intelligent Technologies, Bangkok, Thailand (2000), 307-312.

[11] _ Comparison of interval-valued fuzzy sets, intuitionistic fuzzy sets, and bipolarvalued fuzzy sets, J. Fuzzy Logic Intelligent Systems 14 (2004), 125-129.

[12] F. Marty, Sur une generalization de la notion de groupe, 8th Congress Math. Scandinaves, Stockholm (1934), 45-49.

[13] L. Torkzadeh and A. Ahadpanah, Hyper $M V$-ideals in hyper $M V$-algebras, MLQ Math. Log. Q. 56 (2010), no. 1, 51-62.

[14] L. A. Zadeh, Fuzzy sets, Information and Control 8 (1965), 338-353.

[15] H.-J. Zimmermann, Fuzzy Set Theory and Its Applications, Kluwer-Nijhoff Publishing, 1985. 
Department of Mathematics

HANYANG UNIVERSITY

SeOul 133-791, KoreA

E-mail address: sinchangmyun@hanmail.net 